\title{
Educación Ambiental para el Desarrollo de Capacidades en Comunidades Rurales del Parque Nacional Pico de Orizaba
}

Environmental Education for the Development of Capacities in Rural Communities of the Pico de Orizaba National Park

Landero-Hernández Lourdes ${ }^{1 \bowtie}$, Chamorro-Zarate María de los Ángeles ${ }^{1}$, Domínguez-González Nancy $^{1}$, Viveros-Contreras Rodolfo ${ }^{1}$ y Narave-Flores Héctor Venancio. ${ }^{1}$

${ }^{1}$ Facultad de Biología, Universidad Veracruzana, Circuito Gonzalo Aguirre Beltrán s/n, Zona Universitaria, 91000 Xalapa Enríquez, Veracruz

${ }^{\otimes}$ Autor para correspondencia: $\underline{\text { luluiv@ hotmail.com }}$

Recibido: 08/09/2019

Aceptado: 10/11/2019

\section{RESUMEN}

El Área Natural Protegida Parque Nacional Pico de Orizaba, tiene una superficie de 19, 750 ha, es la montaña con mayor altitud en México con 5, $636 \mathrm{msnm}$. Brinda valiosos servicios ambientales, es una importante área de captación para la recarga de acuíferos, mantiene la red hidrológica superficial de varias cuencas, abasteciendo de agua a más de un millón de personas resguardándolas además, ante fenómenos climáticos extremos. Conservar los bosques de coníferas y páramos de altura de esta zona, contribuye a conservar el único glaciar presente en la montaña. A pesar de ser importante por los servicios que provee, actualmente enfrenta diferentes problemáticas ambientales (caza de fauna silvestre, tala e incendios forestales, por mencionar algunos), que merman las acciones de conservación implementadas. La pérdida de biodiversidad debido principalmente a las actividades realizadas por las comunidades rurales con un alto grado de marginación que se encuentran colindantes, han afectado a especies de gran importancia. En el presente trabajo se aplicaron diferentes estrategias de Educación Ambiental como: capacitaciones, gestión de proyectos, vinculación con otros sectores y con otras comunidades, fortaleciendo las acciones de conservación. Las estrategias se implementaron en dos comunidades: Puerto Nacional en el municipio de Tlachichuca del estado de Puebla y Rancho Nuevo en el municipio de La Perla del estado de Veracruz. Se propició el desarrollo de capacidades de las comunidades, mediante la aplicación de cinco capacitaciones abordando diferentes temas y la creación de grupos de trabajo dando pauta a alternativas laborales que permitan la conservación de la diversidad biológica del parque.

Palabras clave: Área Natural Protegida, servicios ambientales, conservación, biodiversidad.

\begin{abstract}
The Natural Protected Area Pico de Orizaba National Park, has an area of 19,750 ha, is the mountain with the highest altitude in Mexico with 5,636 msnm. It provides valuable environmental services, it is
\end{abstract}


an important catchment area for the recharge of aquifers, it maintains the hydrological surface nertwork of several basins, supplying water to more tan one million people, also protecting them against extreme climatic phenomena. Conserve the coniferous forests and moors of height of this area, contributes to conserve the only glacier present in the mountain. Despite being important for the services it provides, it currently faces different environmental problems (wildlife hunting, logging and forest fires, to name a few), which undermine the conservation actions implemented. The loss of biodiversity due mainly to the activities carried out by the rural communities with a high degree of marginalization that are adjacent, have affected species of great importance. In the present work, different environmental education tools were applied such as: training, project management, linkage with other sectors and with other communities, strengthenning conservations actions. The tools were implemented in two communities: Puerto Nacional in the municipality of Tlachichuca in the state of Puebla and Rancho Nuevo in the municipality of La Perla in the state of Veracruz. The capacity development of the communities was fostered, through the appliction of five trainings addressing different topics and the creation of working groups giving guidelines to work alternatives that allow the conservation of the biological doversity of the park.

Keywords: Protected Natural Area, environmental servicies, conservation, biodibersity.

\section{INTRODUCCIÓN}

La función principal de las Áreas Naturales Protegidas (ANP's) en México, es asegurar la conservación y protección a largo plazo de la biodiversidad y en particular, de las especies y poblaciones amenazadas. Estas áreas, son el ejemplo más claro de la confluencia de procesos biológicos, económicos y sociales.

Bajo esta complejidad y en este contexto, se decretó el 4 de enero de 1937 el Parque Nacional Pico de Orizaba (PNPO), que cuenta con una superficie de 19, 750 ha, comprendidas por cinco municipios tres en el estado de Puebla: Tlachichuca, Chalchicomula de Sesma y Atzizintla y dos en el estado de Veracruz: La Perla y Calcahualco; es la montaña con mayor cota altitudinal en México con una elevación de 5, $636 \mathrm{msnm}$.

Las actividades que se realizan en el parque, están enfocadas a la conservación, vigilancia, manejo, conocimiento y cultura en beneficio de la conservación de los recursos naturales, a través de subsidios (apoyos económicos que una persona recibe a través de un organismo oficial), que otorga la Comisión Nacional de Áreas Naturales Protegidas (CONANP), en cuatro diferentes programas, también se contribuye a la mejora de la calidad de vida de los pobladores de las comunidades rurales cercanas a él, con el fin de promover la conservación de los ecosistemas y su biodiversidad mediante la participación directa de la población. Actualmente esta ANP, enfrenta diferentes problemáticas ambientales (caza de fauna silvestre, tala e incendios forestales), causadas por actividades antrópicas de las comunidades aledañas, que merman las acciones de conservación implementadas.

Conservar los bosques de coníferas y páramos de altura de esta zona, contribuye a conservar el único glaciar presente en la montaña que provee de agua a más de un millón y medio de personas de los municipios cercanos, además las resguarda ante fenómenos climáticos extremos (Comisión Nacional de Áreas Naturales Protegidas, 2015). Si bien es muy importante la 
responsabilidad que deben asumir las instituciones en la protección y mejora del medio ambiente, es también muy necesaria la labor que la sociedad puede realizar. El primer paso para conseguir desarrollar en la población conductas respetuosas con el ambiente es que conozcan la situación actual del problema, facilitándoles información mediante la Educación Ambiental (Red de Autores Ambientales, 2004). El presente trabajo tiene como objetivo contribuir en el desarrollo de capacidades de comunidades rurales, para el cuidado y conservación de los recursos naturales, del PNPO, a través de la aplicación de diferentes estrategias de Educación Ambiental que favorezcan el fortalecimiento de las acciones de conservación que se realizan en parque.

\section{MATERIALES Y MÉTODOS}

Las estrategias de Educación Ambiental abordadas en este trabajo, se basan en actividades encaminadas al desarrollo sostenible, integrando un proyecto de investigación acción-participativa en el que se toman en cuenta las recomendaciones del Plan Veracruzano de Desarrollo (PVD), 2016-2018, la Estrategia de Educación Ambiental para la Sustentabilidad en México de SEMARNAT (Secretaría de Medio Ambiente y Recursos Naturales) y CECADESU (Centro de Educación y Capacitación pata el Desarrollo Sustentable) y el Programa Nacional de Educación para la Sustentabilidad en Áreas
Protegidas de la CONANP; que propician el análisis y la discusión sobre asuntos que requieren atención prioritaria como lo son la educación y comunicación ambiental, teniendo como objetivo la participación activa de las comunidades rurales aledañas al PNPO, ya que son fundamentales las actividades que conlleven a generar/propiciar interacción entre grupos de personas y el medio ambiente.

El trabajo inició con la búsqueda bibliográfica permanente de todos los documentos que proporcionaron información sobre los trabajos de conservación y monitoreo de los recursos naturales realizados en ANP. Las comunidades rurales en las que se aplicaron las actividades, fueron seleccionadas a través de un diagnóstico realizado al personal del PNPO, en el cual se revisaron los antecedentes de las mismas (participación en trabajos de conservación y relación con problemáticas ambientales).

Las COMUNIDADES elegidas fueron: Puerto Nacional en el municipio de Tlachichuca del estado de Puebla POF SU participación en trabajos de conservación, la comunidad de Miguel Hidalgo y Costilla en el municipio de Tlachichuca del estado de Puebla por incidentes con incendios forestales y Rancho Nuevo en el municipio de La Perla del estado de Veracruz, con incidentes de tala clandestina. Posteriormente de manera estratégica en cuatro fases, se realizaron diferentes actividades presentadas en la Tabla 1. 
Tabla 1. Estrategia de Educación Ambiental

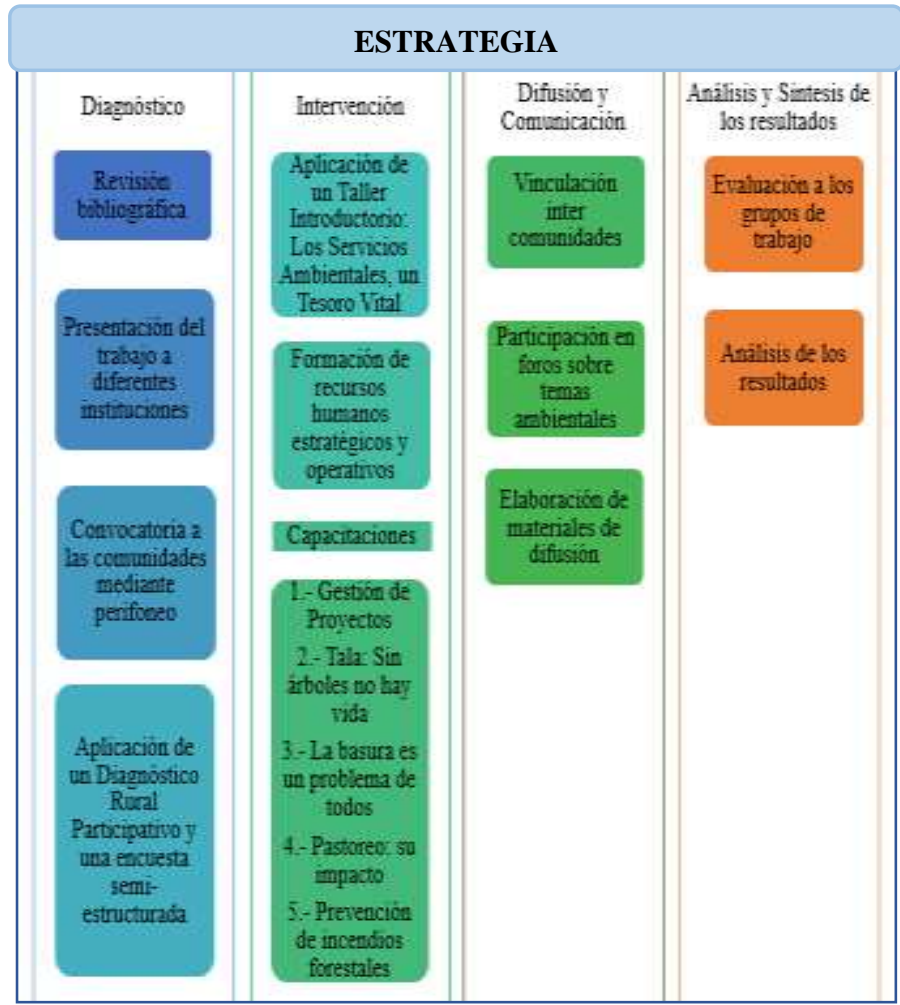

\section{RESULTADOS Y DISCUSIÓN}

Se realizó la presentación del trabajo ante la Dirección del Parque Nacional Pico de Orizaba, las cabeceras municipales; respecto a las comunidades participantes, se les convocó mediante perifoneo. Posteriormente en cada una de ellas se implementó un Diagnóstico Rural Participativo (DRP), en el que se llevaron a cabo diferentes actividades como: dinámica rompehielo, para propiciar la interacción entre los participantes, así como mapas temáticos para conocer los cambios ocurridos en su comunidad respecto a la cobertura forestal y uso de suelo. También se profundizó con el ejercicio de árbol de problemas, para indagar sobre las problemáticas ambientales, sociales y económicas, identificando las causas de las mismas y sus posibles soluciones; Geilfus
(2002) expone que, al aplicar estas dinámicas se puede obtener de manera eficaz y veraz la percepción de la población. La entrevista semiestructurada se realizó de manera individual para que pudieran externar personalmente $y$ de manera verbal, las problemáticas que perciben en su comunidad además de obtener datos sobre las actividades laborales que realizan, Canales (2006) menciona que de esta manera se establece una comunicación interpersonal entre el entrevistador y el sujeto entrevistado, dando pauta a la veracidad de los datos obtenidos. Una vez aplicado el DRP, se ordenaron y analizaron los datos mediante la elaboración de cuadros y se utilizó un generador de nubes de palabras, que permitió poder agrupar las ideas obtenidas de manera ordenada y por ocurrencia con la que aparecieron en la nube de palabras. 
Tabla 2. Problemáticas detectadas mediante el DRP.

\begin{tabular}{|c|c|c|c|c|c|c|c|c|c|c|c|}
\hline \multicolumn{12}{|c|}{ Problemáticas Detectadas } \\
\hline \multicolumn{3}{|c|}{ Dirección del PNPO } & \multicolumn{3}{|c|}{ Miguel Hidalgo y Costilla } & \multicolumn{3}{|c|}{ Rancho Nuevo } & \multicolumn{3}{|c|}{ Puerto Nacional } \\
\hline Econimicos & Sociales & Ambientales & Económicos & Sociales & Ambientales & Ecomómicos & Sociales & Ambientales & Económicus & Sociales & Ambïentales \\
\hline \multirow[t]{7}{*}{$\begin{array}{l}\text { Falte de } \\
\text { Empleos }\end{array}$} & $\begin{array}{l}\text { Falla de } \\
\text { Organización }\end{array}$ & Tala & $\begin{array}{l}\text { Fahta de } \\
\text { Empless }\end{array}$ & $\begin{array}{c}\text { Falta de } \\
\text { Organizacion }\end{array}$ & $\begin{array}{l}\text { Inceedios } \\
\text { foresteles }\end{array}$ & $\begin{array}{l}\text { Falta de } \\
\text { Empleos }\end{array}$ & $\begin{array}{l}\text { Falta de } \\
\text { Organización }\end{array}$ & Tala & $\begin{array}{l}\text { Falta de } \\
\text { Empleoss }\end{array}$ & & $\begin{array}{l}\text { Incendins } \\
\text { forestales }\end{array}$ \\
\hline & $\begin{array}{l}\text { Fala de } \\
\text { Senaibilización }\end{array}$ & $\begin{array}{l}\text { Inceedios } \\
\text { forestales }\end{array}$ & & & Tala & & & $\begin{array}{l}\text { Incendios } \\
\text { forestales }\end{array}$ & & & Pastores \\
\hline & & Pastores & & & Pastores & & & $\begin{array}{c}\text { Basura en las } \\
\text { calles }\end{array}$ & & & Tala \\
\hline & & $\begin{array}{l}\text { Caceria } \\
\text { faxtive }\end{array}$ & & & $\begin{array}{c}\text { Basura en las } \\
\text { barrancas }\end{array}$ & & & Costaminación & & & $\begin{array}{c}\text { Escasez de } \\
\text { agua }\end{array}$ \\
\hline & & $\begin{array}{l}\text { Turizmo } \\
\text { irrespensable }\end{array}$ & & & Caceria furtiva & & & & & & \\
\hline & & Plages & & & $\begin{array}{l}\text { Escasez de } \\
\text { agua }\end{array}$ & & & & & & \\
\hline & & & & & $\begin{array}{l}\text { Desechos de } \\
\text { perros }\end{array}$ & & & & & & \\
\hline
\end{tabular}

Se identificaron cuatro principales problemáticas ambientales, una social y una económica, presentadas en la Tabla 2; funcionando ésta herramienta, como una alternativa para recuperar la percepción de las personas participantes. Tomando como referencia los resultados obtenidos en la tabla, se dieron prioridad a cuatro problemáticas ambientales en las que coincidieron todos los casos: tala, incendios forestales, pastoreo y la basura; para el caso de las problemáticas sociales y económicas se detectó que, no hay organización interna en las comunidades y falta de empleos duraderos.

Ya que se identificaron las problemáticas se diseñaron y aplicaron diferentes capacitaciones para que se brindara información sobre el impacto ambiental que tienen las actividades antrópicas que realizan. La fase de intervención dio inicio con la implementación de un taller introductorio: "Los Servicios Ambientales: Un Tesoro Vital", el cual permitió la interacción de las personas de las comunidades propiciando el interés sobre la temática ambiental.

El taller introductorio dio pie a la conformación de tres grupos de trabajo (uno por cada comunidad), quienes recibieron en diferentes momentos las capacitaciones diseñadas $\mathrm{y}$ adecuadas para los grupos: 1. Gestión de Proyectos ante la CONAFOR y CONANP; 2. Tala: Sin árboles no hay vida; 3. La basura es un problema de todos; 4. Pastoreo: su impacto y 5. Prevención de incendios forestales. En cada una de las capacitaciones se proyectaron imágenes y videos, que dieron apertura a pláticas de reflexión en donde las personas compartieron sus experiencias, y así, se promovió en los grupos de trabajo la revaloración de las acciones que realizan y que afectan al ecosistema del PNPO. Para las últimas dos capacitaciones, se decidió integrar a representantes de las brigadas de conservación y vigilancia establecidas por el PNPO, quienes constantemente se enfrentan al combate de contingencias ambientales, permitiendo el intercambio de experiencias y la 
vinculación con otras comunidades. En total se implementaron tres talleres introductorios y 13 capacitaciones en las tres comunidades participantes, conformando solo dos grupos de trabajo el primero en la comunidad de Rancho Nuevo y el segundo en la comunidad de Puerto Nacional.

El diseño y elaboración de los materiales de difusión se implementaron como parte de una estrategia de comunicación que se realizó en dos secciones, la primera: el diseño de un logotipo de Educación Ambiental en el PNPO, para crear identidad y pertenencia en los grupos de trabajo, el diseño de un calendario en el que se plasmaron algunas temáticas abordadas en las capacitaciones y por último el diseño de playeras para impresión, fomentando localmente con estos materiales la sensibilización al cuidado de los recursos, de su entorno y del PNPO. En la segunda sección se diseñó para difusión general, un Sitio Web, en donde se integró información sobre los programas de conservación que se han realizado en el parque, así como la participación de las comunidades, también se gestionó ante la Dirección de Área Biológico-Agropecuaria de la Universidad Veracruzana, la participación del Laboratorio Multimedia X-Balam de la Facultad de Biología Xalapa, en la filmación, edición y producción de un documental titulado "Parque Nacional Pico de Orizaba: Una Joya de la Naturaleza", en el que se difunde información diversa y de alto valor sobre las acciones de conservación y/o restauración que se realizan en el parque y la participación social que se ha obtenido como resultado.

Como Weber (1975); CNUMAD (1992); Beck (1998); Robertson (1998); Leff (2004) y NovoVillaverde (2011), lo mencionan en diferentes obras la especialización de conocimientos para construir una racionalidad ambiental, incorporando las relaciones entre la sociedad y la naturaleza, son una vía de replanteamiento de las actividades antrópicas realizadas por las comunidades cercanas.

Traduciendo la información proporcionada mediante las capacitaciones, en la pertenencia y conservación de los recursos naturales, permitiendo así, la articulación eficiente de los programas y proyectos de conservación futuros con miras en la sostenibilidad, con la participación social que contribuyan en un trabajo colectivo a la conservación de los recursos naturales del Parque Nacional Pico de Orizaba.

\section{CONCLUSIONES}

Es necesario implementar la Educación Ambiental como un proceso de comunicación educativa, que no busque simplemente transmitir información y mensajes, sino educar y formar a los grupos de trabajo a través de procesos sistemáticamente organizados que influyan en el cambio de percepción y actitudes. Es importante fomentar una comunicación efectiva contextualizada y multimodal para atender las necesidades de información y formación de las comunidades.

Se debe promover una educación más participativa y menos sensacionalista, que ponga en marcha su visión interdisciplinaria, construyendo un diálogo que facilite la articulación y apropiación de la información en miras de una sociedad proambiental y sostenible.

Las instituciones deben buscar una relación estrecha con las poblaciones aledañas al parque, ya que en ellas se centra el éxito de las acciones de conservación, éste será quizá, el reto a 
Landero-Hernández et al., 2019

enfrentar en la elaboración de un Programa de Educación Ambiental para el Parque Nacional Pico de Orizaba, ya que podría ser la herramienta que toque las fibras más sensibles de las comunidades, pasando de comunidades marginadas a comunidades autogestoras, para conservar los recursos naturales del PNPO.

\section{LITERATURA CITADA}

Beck, U., 1998. La Sociedad del Riesgo: Hacia una nueva modernidad. Edición en Castellano. Ediciones Paidós Ibérica, S.A., ISBN: 84-493-0406-7. Santa Perpétua de Mogoda, Barcelona, España.

Canales-Cerón, M., 2006. Metodologías de la investigación social. Santiago, LOM Ediciones.

Comisión Nacional para el Conocimiento y Uso de la Biodiversidad, 2017. La Integración de la Biodiversidad para el Bienestar. CONABIO. Revista Biodiversitas Núm. 131 Marzo-Abril, 2017, ISSN: 18701760, México, D.F.

Comisión Nacional de Áreas Naturales Protegidas (CONANP), 2011. Parque Nacional Pico de Orizaba, Dirección de la Región Planicie Costera y Golfo de México.

http://regiongolfodemexico.conanp.gob. mx/pico_orizaba.php.

Comisión Nacional de Áreas Naturales Protegidas (CONANP), 2015. "Conservación de los Mamíferos del Parque Nacional Pico de Orizaba". Informe técnico. Programa de Especies en Riesgo (PROCER), Parque Nacional Pico de Orizaba, México.
Comisión Nacional de Áreas Naturales Protegidas (CONANP), 2015. "Monitoreo y Conservación de Especies". Informe técnico. Programa de Conservación Para el Desarrollo Sostenible (PROCODES), Parque Nacional Pico de Orizaba, Comisión Nacional de Áreas Naturales Protegidas, Atotonilco, Calcahualco, Veracruz, México.

Comisión Nacional de Áreas Naturales Protegidas (CONANP), 2016. "Capacitación: Conocimiento y Aplicación de Herramientas y Metodologías para el Monitoreo de Mamíferos Terrestres en el Parque Nacional Pico de Orizaba". Informe técnico. PROMANP- Componente Vigilancia Comunitaria, Parque Nacional Pico de Orizaba, México.

CNUMAD, 1992. Conferencia de las Naciones Unidas Sobre Medio Ambiente y Desarrollo, Antecedentes Históricos, Capítulo 4, p. 25-46.

Geilfus, F., 2002. 80 Herramientas para el Desarrollo Participativo: diagnóstico, planificación, monitoreo y evaluación. Instituto Interamericano de Cooperación para la Agricultura (IICA). ISBN13: 99923-7727-5, San José, Costa Rica.

Leff, E., 2004. Racionalidad Ambiental, la reapropiación social de la naturaleza, Ed. Siglo XXI, México, p. 244.

Novo-Villaverde, M., 2011. La Educación Ambiental en Tiempos de Crisis. Trasatlántica de Educación. , ISSN 18706428, No. 9, p. 7-14.

Red de Autores Ambientales, 2004. "Sensibilización Ambiental”. Boletín 
Landero-Hernández et al., 2019

Informativo. Recuperado el 13 de Octubre de 2016 en: http://www.magrama.gob.es/es/calidady-evaluaciónambiental/temas/red-deautoridades-ambientales-raa/boletinsenssib_tcm7-15079.pdf

Robertson, R., 1998. Identidad nacional y globalización: falacias contemporáneas.
Revista Mexicana de Sociología. Instituto de Investigaciones Sociales. Universidad Nacional Autónoma de México. Vol. 60, núm. 1, p. 3-19.

https://doi.org/10.2307/3541253

Weber, M., 1975. Economía y Sociedad, Fondo de Cultura Económica, Tomo I, México, 1975, Pp. 36.

Copyright (c) 2019 L ourdes L andero-Hernández, Maria de los Ángeles Chamorro-Zarate, Nancy Dominguez-González, Rodolfo ViverosContreras, Venancio Narave-Flores

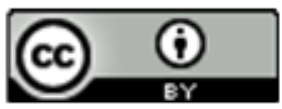

Este texto está protegido por una licencia licencia CreativeCommons 4.0.

Usted es libre para Compartir — copiar y redistribuir el material en cualquier medio o formato- y Adaptar el documento — remezclar, transformar y crear a partir del material- para cualquier propósito, incluso para fines comerciales, siempre que cumpla la condición de:

Atribución: Usted debe dar crédito a la obra original de manera adecuada, proporcionar un enlace a la licencia, e indicar si se han realizado cambios. Puede hacerlo en cualquier forma razonable, pero no de forma tal que sugiera que tiene el apoyo del licenciante o lo recibe por el uso que hace de la obra.

Resumenclelicencia - Textocompletodelalicencia 